\title{
Inferences about mechanisms that mediate pattern and flicker sensitivity
}

\author{
EDWARD M. BRUSSELL, CHARLES W. WHITE, PARDO MUSTILLO, \\ and OLGA OVERBURY \\ Concordia University, Montreal, Quebec, Canada
}

\begin{abstract}
Visual sensitivity to a flickering, sinusoidal grating was assessed in multiple sclerosis patients and control observers. It was found that losses in spatial contrast sensitivity exhibited by patients were independent of the rate at which the grating was flickered. However, more often than for the controls, the detection criterion for the patients was based upon seeing a pattern rather than flicker. These findings are interpreted in light of the recent controversy concerning whether there is more than one set of neural mechanisms responsible for processing information about pattern and flicker. It is concluded that the data are consistent with the view that the human visual system contains partially independent channels.
\end{abstract}

The rationale for this research stems from two converging sources. The first is speculation, based upon psychophysical data, that the human visual system contains separate channels for processing information about pattern and flicker (Bodis-Wollner \& Hendley, 1979; Keesey, 1972; King-Smith \& Kulikowski, 1975; Kulikowski \& Tolhurst, 1973; Tolhurst, 1973). The idea derives from Keesey's (1972) observation that different thresholds can be measured for detecting a pattern and for detecting flicker. Kulikowski and Tolhurst (1973) confirmed this finding using grating patterns, and reported that whether a pattern or flicker threshold was lower depended upon the spatial frequency that was presented; flicker was seen at lower contrasts when the spatial frequency was low, and pattern was seen first when the spatial frequency was high. Although this type of evidence seems, on the surface, to be quite direct and compelling, it has been seriously questioned in recent years (Burbeck, 1981; Derrington \& Henning, 1981; Kelly, 1982).

The second line of research that underlies this study involves attempts to apply psychophysical techniques to aid in the diagnosis and monitoring of visual disorders (Enoch \& Williams, 1983). These techniques have been used to assess residual spatial sensitivity (e.g., Bodis-Wollner, 1972; Regan, Silver, \& Murray, 1977; Zimmern, Campbell, \& Wilkinson, 1979), as well as temporal sensitivity (e.g., Brussell,

This report is based on a paper delivered at the annual meeting of the Association for Research in Vision and Ophthalmology, Sarasota, Florida, May 1982 . The study was supported by grants from the Natural Sciences and Engineering Research Council of Canada (Nos. A9937 to E.M.B. and A7482 to C.W.W.). Reprint requests may be directed to: E. M. Brussell, Department of Psychology, Concordia University, 1455 de Maisonneuve Boulevard West, Montreal, Quebec, Canada H3G 1 M8.
White, Bross, Mustillo, \& Borenstein, 1981, 1982; Daley, Swank, \& Ellison, 1979; Galvin, Regan, \& Heron, 1976) in various patient populations. However, there have been few, if any, reports of measurements of spatial and temporal sensitivity in the same patient, with the same stimuli (i.e., spatiotemporal sensitivity).

From a basic research point of view, measuring spatiotemporal sensitivity in the diseased visual system can shed light on normal functioning in a way analogous to that of studying illusions. In the latter case, one can formulate testable hypotheses about how the visual system functions by considering how the normal system, conceptualized in a particular way, might be "tricked" into producing an illusion. When studying the diseased visual system, one may make inferences about normal functioning by carefully considering the types of visual loss that are observed in the context of the known physiological consequences of the disorder. Thus, questions about how many mechanisms mediate pattern and flicker sensitivity can be pursued by studying patient populations. For example, differential spatial and temporal loss within an observer might suggest the existence of different neural pathways that mediate spatial and temporal vision. The purpose of the present experiment was to empirically explore this line of reasoning.

\section{METHOD}

\section{Observers}

Fifteen individuals diagnosed as "clinically definite" multiple sclerosis (MS) patients and 11 control observers participated in the experiment. All control observers had corrected or uncorrected $20 / 20$ visual acuity, and all patients had corrected acuity no worse than 20/30 (acuity measured with the Keystone Visual Skills test). No patient suffered from or had a history of a visual disorder other than optic neuritis, and no control observer either suffered from or had a history of any ophthalmological disorder. All ob- 
servers were asked to wear their best correction for far vision during the experiment.

\section{Apparatus and Stimuli}

Gratings with a sinusoidal luminance profile were generated on a cathode ray tube (Hewlett-Packard 1310A with a P15 phosphor) according to the method outlined by Campbell and Green (1965). Under conditions in which the contrast of the grating was modulated in time (on-off sinusoidal flicker), an additional function generator was used to modify the input to the Z-axis amplifier of the CRT. The frequency and amplitude of the Z-axis input were controlled by a PDP-11/10 computer. At a viewing distance of $100 \mathrm{~cm}$, the test gratings appeared in a square field whose sides subtended $4 \mathrm{deg}$ of visual angle and whose mean luminance was $1.5 \mathrm{~cd} / \mathrm{m}^{2}$ (measured with a Spectra Spot Meter).

\section{Procedure}

In order to take into account the physical disabilities often exhibited by MS patients, a psychophysical procedure was adopted that minimized the testing time. Each trial began with the presentation of a subthreshold grating whose contrast was increased in steps of $.05 \mathrm{log}$ units. An increase in contrast occurred every $250 \mathrm{msec}$ or after two complete flicker cycles, whichever was longer, and continued until the observer pressed a response key indicating that detection had occurred. Before a testing session began, a simple reaction time was assessed to a 2.8-cycle-perdegree (cpd) grating that was approximately $0.1 \log$ unit above threshold. In order to allow more valid comparison among patients and between groups, the computer used this reaction time in specifying the contrast of the grating that was displayed at an estimated time of detection, rather than at the time at which the response key was pressed. In addition to the manual response, the observer also stated whether detection was based upon seeing a pattern or unpatterned flicker.

Various combinations of spatial frequencies, ranging from 1 to $13.5 \mathrm{cpd}$ in .75 -octave-unit steps, and temporal frequencies, ranging from 1 to $32 \mathrm{~Hz}$ in 1-octave-unit steps (plus $0 \mathrm{~Hz}$ ), were presented. For a given flicker rate, the six spatial frequencies were tested in a random order. For each spatial frequency, at least seven trials were presented, the first two of which were considered practice. Interspersed among the seven trials were three additional ones during which no grating was presented. The inclusion of these catch trials allowed the assessment of a false alarm rate to monitor the stability of a given observer's response criterion. A block of trials continued until seven responses were made (not including the catch trials) or until seven gratings were undetected at the highest contrast that was presented $(0.7$, the upper linear region of the CRT).

All viewing was monocular, and both eyes were tested. No fixation point was provided and observers were instructed that during a trial they should shift their gaze around the lit window within which a grating would appear. This was done in order to reduce the likelihood of the development of afterimages.

\section{RESULTS AND DISCUSSION}

The false alarm rates were quite low for both the patients and controls (3.6\% and $1.3 \%$, respectively). This indicated that a consistently conservative decision criterion was adopted by all observers, even though there was no inducement to that effect as part of the procedure.

Figure 1 presents contrast sensitivity (the reciprocal of the contrast threshold for detecting the presence of a grating) as a function of the number of cycles in the grating per degree of visual angle (spatial frequency) and flicker rate (temporal frequency). Panel $A$ de-

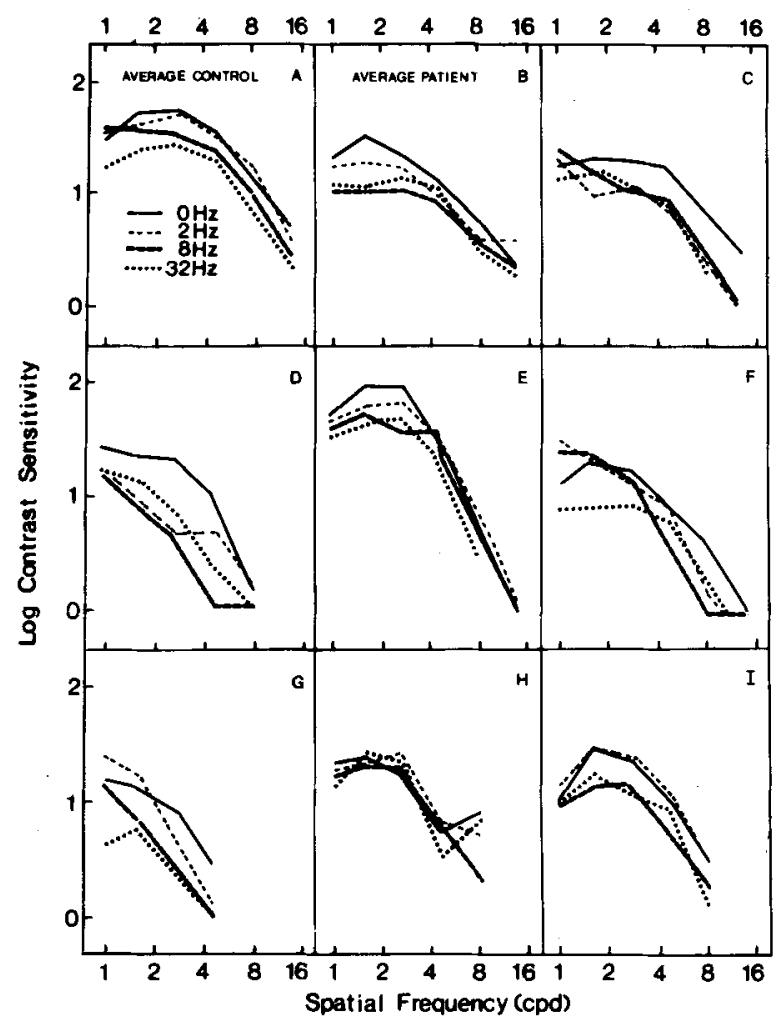

Figure 1. Log contrast sensitivity to a grating stimulus for varlous combinations of apatial and temporal frequencies. Panels $A$ and $B$ show the pooled functions for the control and patient observers, respectively. Panels $\mathbf{C}$ through I present monocular data for Individual patients.

picts the pooled data from the control group, and Panel B presents the pooled data from the patient group. In these two panels, each data point represents the mean log contrast sensitivity only for those observers who were able to detect the grating. Although there were only a few instances in which control observers could not detect the grating at even the highest contrast that was used, there were many patients for whom this did occur, especially at the higher spatial frequencies (e.g., Panel G). Thus, every patient was included in the pooled functions of Panel B, but not every pooled data point included all of the patients.

Compared with the control functions, the average patient typically exhibited reduced contrast sensitivity. However, averaging data across patients does not provide an informative way of presenting the results. The reason is that MS patients can show deficits at any spatial frequency (Bodis-Wollner, Hendley, Mylin, \& Thornton, 1979; Regan, Raymond, Ginsburg, \& Murray, 1981; Zimmern et al., 1979) - a fact that is lost in the smoothed, averaged functions, but preserved in the individual patient functions, examples of which are shown in Panels C-I. The most notable characteristic of these patient functions is the ten- 
dency for the general abnormality in contrast sensitivity for any individual to be independent of the temporal frequency at which the grating was flickered. For example, the patient whose data is presented in Panel G shows a rapid decline in sensitivity at all flicker rates. Also, although in Panel E contrast sensitivity is better than average at low spatial frequencies, the high-frequency roll-off is noticeably steeper at all temporal frequencies than in the corresponding portions of the control functions.

This finding immediately suggests that the same diseased pathways were carrying the information about the spatial and temporal characteristics of the stimulus. The reason is that if flicker and pattern information were transmitted through the same neural pathways, then an aberration in contrast sensitivity that is observed with a stationary grating should also be present when the grating is flickered. However, this interpretation must, at the very least, be seriously questioned in light of the data presented in Figure 2.

The six panels in this figure illustrate the proportion of responses for which detection was based upon the report of seeing a pattern rather than a flicker. At the higher temporal frequencies (i.e., 16 and $32 \mathrm{~Hz}$ ), all observers tended to say that they saw a pattern at threshold. This is consistent with other findings that indicate that, under the low-luminance conditions of our experiment, flicker sensitivity is expected to be extremely poor, especially at higher temporal frequencies (Kelly, 1961). However, at flicker rates less than $16 \mathrm{~Hz}$, there was a consistent trend for the pa-

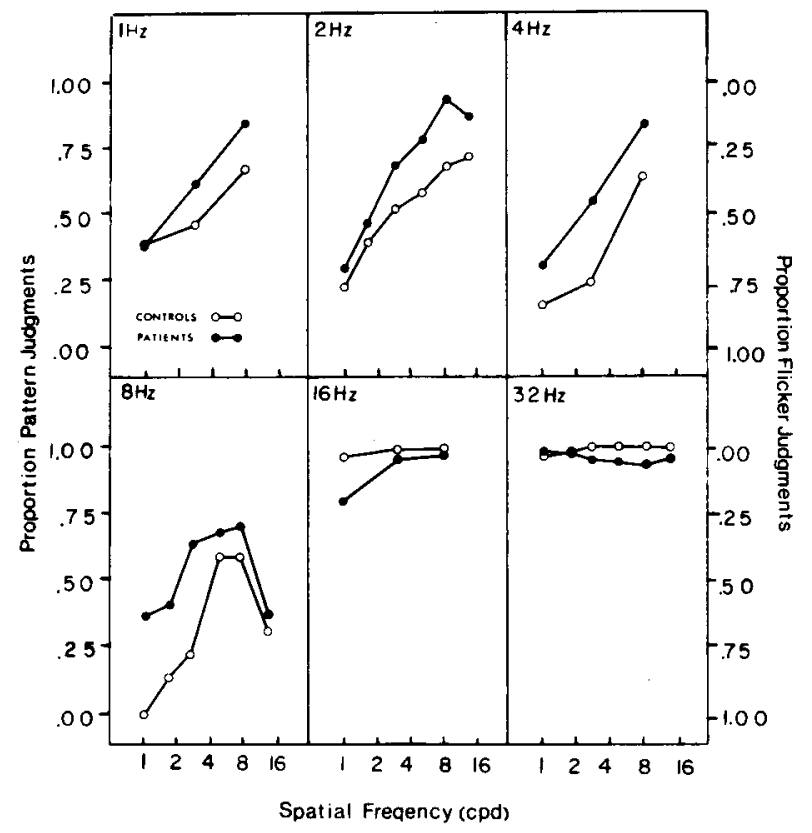

Figure 2. The proportion of pattern (ficker) judgments as a function of spatial frequency for the patient (filled circles) and control (open eirciea) groups. Each panel presents the data for a different temporal frequency. tients to state, more often than the controls, that they saw a pattern rather than flicker at the detection threshold. The problem concerns how there can be differential responding with respect to detection being based upon pattern and flicker, if the same diseased pathways are carrying the information about both pattern and flicker.

One possibility lies at the heart of the recent controversy regarding the evidence favoring the view that separate mechanisms exist for processing information about pattern and flicker. Much of this evidence stems from data indicating that different pattern and flicker thresholds can be measured in a contrast sensitivity paradigm (e.g., Kulikowski \& Tolhurst, 1973). However, Burbeck (1981) has recently and effectively argued that independent contrast thresholds for detecting flicker and pattern do not necessarily imply separate visual mechanisms for processing flicker and pattern information. Rather, the independent thresholds may reflect separate psychophysical response criteria for stating that detection was based upon seeing flicker or a pattern.

Although we agree with this point in general, we do not think that the data presented in Figure 2 are subject to this criticism for a number of reasons. The first is that we did not impose a response criterion on our observers. That is, we did not block the trials with respect to pattern and flicker detection. Rather. we allowed the observers to establish their own criteria and freely report whether on any given trial they felt that detection was based upon seeing pattern or flicker. Second, if the tendency to say "pattern" or "flicker" primarily reflects a response bias, then one would be hard pressed to explain why at threshold MS patients have a greater bias towards saying "pattern" than do the controls. Furthermore, given the reasonable assumption that recognizing a pattern reflects a more difficult decision than detecting flicker (Burbeck, 1981), one would expect the patients to base their response on a decision that they saw flicker more often than the controls would.

We therefore suggest that the most parsimonious way of accounting for this stronger tendency for the patients to say "pattern" is to assume that (1) there are different visual mechanisms for processing information about flicker and pattern, and (2) the patients depended on the output of the pattern mechanism for detecting the grating more often than did the controls. The reason that the patients relied more heavily on the pattern mechanism may well be that MS induces a relatively greater functional impairment within the flicker pathways than within the pattern pathways. This should not be interpreted to mean that there is, for example, a tendency for greater demyelination to occur in the flicker pathways. Rather, any degree of demyelination may have a more disruptive effect in the flicker pathways than in the pattern pathways. 
According to this interpretation, it is not surprising that in Figure 1 the general impairment in contrast sensitivity did not depend on the rate at which a grating was flickered. The reason would be that the spatial contrast sensitivity functions primarily reflect the operation of the visual mechanism that is relatively more sensitive: the pattern mechanism. However, it also should be noted that this interpretation does not demand the postulation of entirely separate mechanisms for pattern and flicker, and is consistent with recent speculation that they may not be completely independent (Essock \& Lehmkuhle, 1982; Murray, MacCana, \& Kulikowski, 1983). It only requires that at some point in the system they be separate so that contrast sensitivity can be dominated by the one that is less impaired.

\section{REFERENCES}

Bodis-Woltner, I. (1972). Visual acuity and contrast sensitivity in patients with cerebral lesions. Science, 178, 769-771.

Bodis-Wollne r, I., \& Hendley, C. D. (1979). On the separability of two mechanisms involved in the detection of grating patterns in humans. Journal of Physiology (London), 291, 251-263.

Bodis-Wollner, I., Hendley, C.D., Mylin, L. H., \& Thornton, J. (1979). Visual evoked potentials and the visuogram in multiple sclerosis. Annals of Neurology, 5, 40-47.

Brusseld, E. M., White, C. W., Bross, M., Mustillo, P., \& Borenste in, M. (1981/1982). Multi-flash campimetry in multiple sclerosis. Current Eye Research, 1, 671-677.

Burbeck, C. A. (1981). Criterion-free pattern and flicker thresholds. Journal of the Optical Society of America, 71, 1343-1350.

Campeell, F. W., \& Green, D. G. (1965). Optical and retinal factors affecting visual resolution. Journal of Physiology (London), 181, 576-593.

Daley, M. L., Swank, R. L., \& Ellison, C. M. (1979). Flicker fusion thresholds in multiple sclerosis: A functional measure of neurological damage. Archives of Neurology, 36, 292-295.
Derainaton, A. B., \& Henning, C. B. (1981). Pattern discrimination with flickering stimuli. Vision Research, 21, 597-602.

Enoch, J. M., \& Williams, R. A. (1983). Development of clinical tests of vision: Initial data on two hyperacuity paradigms. Perception \& Psychophysics, 33, 314-322.

Essock, E. A., \& LeнmRUhLe, S. (1982). The oblique effects of pattern and flicker sensitivity: Implications for mixed physiological input. Perception, 11, 441-455.

Galvin, R. J., Regan, D., \& Henon, J. R. (1976). Impaired temporal resolution of vision after acute retrobulbar neuritis. Brain, 99, 255-268.

KeEsey, U. T. (1972). Flicker and pattern detection: A comparison of thresholds. Journal of the Optical Society of America, $62,446-448$.

KeLLY, D. H. (1961). Visual responses to time dependent stimuli. I. Amplitude sensitivity measurements. Journal of the Optical Society of America, 51, 422-429.

KeLLY, D. H. (1982). Fourier components of moving gratings. Behavior Research Methods \& Instrumentation, 14, 435-437.

King-Smith, P. E., \& Kulikowski, J. J. (1975). Pattern and flicker detection analysed by subthreshold summation. Journal of Physiology (London), 249, 519-548.

KulikowskI, J. J., \& Tolhurst, D. J. (1973). Psychophysical evidence for sustained and transient detectors in human vision. Journal of Physiology (London), 232, 149-162.

Murany, I., MacCana, F., \& Kulikowski, J. J. (1983). Contribution of two movement detecting mechanisms to central and peripheral vision. Vision Research, 23, 151-159.

Regan, D., Raymond, J., Ginsbura, A. P., \& Murray, T. J. (1981). Contrast sensitivity, visual acuity and the discrimination of Snellen letters in multiple sclerosis. Brain, 104, 333-350.

Regan, D., Silver, R., \& Murray, T. J. (1977). Visual acuity and contrast sensitivity in multiple sclerosis-hidden visual loss. Brain, 100, 563-579.

Tolnunst, D. J. (1973). Separate channels for the analysis of the shape and the movement of a moving stimulus. Journal of Physiology (London), 231, 385-402.

Zimmern, R. L., Campbell, F. W., \& Wilkinson, I. M. S. (1979). Subtle disturbances of vision after optic neuritis elicited by studying contrast sensitivity. Journal of Neurology, Neurosurgery, and Psychiatry, 42, 407-412.

(Manuscript received August 15, 1983; accepted for publication February 17, 1984.) 